\title{
APPLICATION OF INPUT-STATE OF THE SYSTEM TRANSFORMATION FOR LINEARIZATION OF SELECTED ELECTRICAL CIRCUITS
}

\author{
Andrzej Zawadzki — Sebastian Różowicz *
}

\begin{abstract}
The paper presents a transformation of nonlinear electric circuit into linear one through changing coordinates (local diffeomorphism) with the use of closed feedback loop. The necessary conditions that must be fulfilled by nonlinear system to enable carrying out linearizing procedures are presented. Numerical solutions of state equations for the nonlinear system and equivalent linearized system are included.
\end{abstract}

K e y w ords: nonlinear electrical circuits, linearization, feedback linearization, local diffeomorphism, Lie algebras, differential geometric approach

\section{INTRODUCTION}

The actual physical systems exhibit the features of nonlinearity and additionally have time-varying parameters. The study of such systems boils down to the analysis of mathematical model which in general case is nonlinear. The analysis of nonlinear systems, especially in dynamic states, is a very difficult task of circuit theory. In most cases, there is no analytical solution of the problem (which is frequently sought) and the information about the current flow and voltage distribution can be obtained using the methods of numerical integration. As shown in [1-4], although they are universal and applicable to any number of differential equations, they generate a numerical solution (compiled in the form of tables, graphs, etc). Therefore, in the search for analytical solutions the transformation of nonlinear description into a linear one by linearization (ensuring the equilibrium of the system dynamics) is very useful in solving practical problems relating to the behaviour of nonlinear circuits.

The modern theory of nonlinear systems, in particular the geometric approach, has acquired major significance in the linearization of nonlinear systems. The application of geometric approach to solve nonlinear problems initiated by Brockett in [5] was used in control theory with observability and controllability of the systems $[6,7]$ taken into account. The methods of differential geometry allowed the development of efficient techniques for the analysis and synthesis of such systems. The series of publications [8-12], which considered the problem of nonlinear transformation of a linear system by changing the coordinates (local diffeomorphism) and using feedback [10-19] considerably contributed to the development of the techniques mentioned.

On the basis of papers mentioned it can be concluded that although geometric methods are mainly applied in control theory, they can be also used in other areas, eg, in the theory of electrical circuits. Such attempts have already been made (for example, [20-23]), but they are too few and not exhaustive enough. Since the presented issues are still valid, we attempt to use geometrical methods in the theory of electrical circuits. It seems that the results presented can easily find other applications.

We consider nonlinear systems (modelling input- state of the system) given by

$$
\frac{\mathrm{d} \mathbf{x}(t)}{\mathrm{d} t}=\boldsymbol{f}(\mathbf{x}(t))+\sum_{i=1}^{m} \boldsymbol{g}_{i}(\mathbf{x}(t)) \mathbf{u}_{i}(t), \mathbf{x} \in \mathbf{R}^{n},
$$

where $t$ - time, $\mathbf{x}(t)$ - vector of state variables, $\boldsymbol{u}_{i}(t)$ - input vector, $\boldsymbol{f}$ and $\boldsymbol{g}_{1}, \boldsymbol{g}_{2}, \ldots, \boldsymbol{g}_{m}$ are smooth vector fields defined on the $M=\mathbf{R}^{n}$ manifold, known as the state space. Methods of differential geometry allow linearization (decoupling and decomposition) of nonlinear systems (1) to the following linear form

$$
\frac{\mathrm{d} \boldsymbol{z}(t)}{\mathrm{d} t}=\mathbf{A} \boldsymbol{z}(t)+\mathbf{B} \mathbf{v}(t)
$$

where $\boldsymbol{z}(t)$ and $\vec{v}(t)$ are new state and input vectors and $\mathbf{A}$ and $\mathbf{B}$ are matrices of proper dimensions. Equation (2) is, of course, a linear equation shown in the new state space with variables $\boldsymbol{z}(t)$. It is therefore, possible to analyse such a model using methods known from the theory of linear systems, and then transfer the results to the nonlinear system (1) by means of inverse transformation $S^{-1}(x)$.

Linear models (2) of nonlinear systems (1) are the result of linearizing transformation transforming a nonlinear system by changing the coordinates in the state space. The change consists in replacing the original state variables $\mathbf{x}(t)$ by new variables $\boldsymbol{z}(t)$ which describe the system in new state space. The linearizing effect of such a

* Faculty of Electrical Engineering, Automatics and Computer Science, Kielce University of Technology, 25-314 Kielce, al. Tysiąclecia Państwa Polskiego 7, Poland, a.zawadzki@tu.kielce.pl, s.rozowicz@tu.kielce.pl 
transformation - transforming a nonlinear system by a change of coordinates in the state space can be represented as follows

$$
S(\mathbf{x}): \mathbf{x}(t) \Longrightarrow \mathbf{z}(t)
$$

where $S(\mathbf{x})$ - linearizing transformation, $\boldsymbol{z}(t)$ a new state vector. The systems which cannot be globally linearized and are transformed only into quasilinear systems can be further linearized by the feedback. In this case, a combination of linearization by transformation of state variables and input transformation $\mathbf{u}(t)$ using a feedback is applied. As the result of the transformation involving the change of coordinates and the introduction of the feedback, the state vector in a new coordinate system can be illustrated as

$$
\boldsymbol{z}=S(\mathbf{x})+\text { feedback }
$$

The problems associated with the analysis of nonlinear systems subjected to linearizing transformations can be divided into the following two groups of tasks:

1) linearization of a nonlinear system by transformation of the coordinate system, for which we can further distinguish:

a. transformation input-state of the system,

b. transformation input-output of the system.

2) linearization by transformation of the coordinate system and feedback where we can distinguish:

a. transformation input-state of the system obtained by the feedback,

b. transformation input-output of the system obtained by the feedback.

Linearization of nonlinear generator by transformation input-state of the system (task 1a) is presented in [23], while for nonlinear circuit is presented in [24]. Our aim in this paper is to deal with linearization of a group of nonlinear electrical circuits by feedback (task $2 \mathrm{a}$ ). The main examples are the systems describing indefinite state in electrical circuits containing elements that collect energy (eg systems with RLC elements), or those described by Van der Pol or Lotka-Volterra nonlinear equations.

For the construction of linearizing transformation (4) the elements of Lie algebra (Lie derivative and the properties of group operation-Lie brackets) [25-27] are applied. The paper is organized as follows. Chapter 2 provides basic definitions and theorem concerning the conditions to be met by nonlinear system to perform the linearizing procedures. The elements of Lie algebra, used in the construction of a new base of state space are included. The effective transformation of mathematical model of nonlinear electrical circuit illustrating the operation of the linearization is given in Chapter 3. Conclusions and comments are presented in Chapter 4 . The paper ends with a list of references.

\section{PRELIMINARIES}

Some basic definitions and concepts of differential geometry are presented. Attempts have been made to discuss them in a simplified and compact form. More detailed information can be found in the references [1012, 25-30].

In the analysis of nonlinear systems the operation involving smooth vector fields $\boldsymbol{f}$ and $\boldsymbol{g}$ defined on an open set $M$ of space deserves special attention. The result of the operation (defined below) is a new smooth vector field.

DEFINITION 1. Let $\boldsymbol{f}$ and $\boldsymbol{g}$ be vector fields defined on the manifold $M=\mathbf{R}^{n}$. Lie brackets of the vector field are called the third vector field, defined by

$$
[\boldsymbol{f}, \boldsymbol{g}]=\frac{\partial \boldsymbol{g}}{\partial \mathbf{x}} \circ \boldsymbol{f}-\frac{\partial \boldsymbol{f}}{\partial \mathbf{x}} \circ \boldsymbol{g}=(\nabla \otimes \boldsymbol{g}) \circ \boldsymbol{f}-(\nabla \otimes \boldsymbol{f}) \circ \boldsymbol{g},
$$

where $\nabla$ - Hamilton operator, o-scalar product, $\nabla \otimes \mathbf{g}$ and $\nabla \otimes \boldsymbol{f}$ are gradients of vector fields $\vec{f}$ and $\boldsymbol{g}$. Lie brackets can be written in a compact form (used in the subsequent parts of the paper) as $\operatorname{ad}_{\boldsymbol{f}} \boldsymbol{g}$.

The next operation is Lie derivative comprising realvalued function $\mathrm{h}$ and vector field $\mathrm{f}$ defined on manifold $M$ of $\mathbf{R}^{n}$ space. The result of the operation is a smooth real-valued function defined for each $\mathbf{x}$ from $M$ set as follows:

Definition 2. Let mapping $h: \mathbf{R}^{n} \rightarrow \mathbf{R}^{n}$ be a smooth scalar function $n$ of $\mathbf{x}=\left(x_{1}, x_{2}, \ldots, x_{n}\right)^{\top} \in \mathbf{R}$ variables and $\boldsymbol{f}: \mathbf{R}^{n} \rightarrow \mathbf{R}^{n}$ vector field defined on manifold $M=\mathbf{R}^{n}$, then Lie derivative of scalar function $h(\mathbf{x})=h\left(x_{1}, x_{2}, \ldots, x_{n}\right)$ directed towards $\vec{f}$ field is the scalar function given by

$$
L_{\boldsymbol{f}} h=\frac{\partial h}{\partial \mathbf{x}} \circ \boldsymbol{f}=\sum_{i=1}^{n} \frac{\partial h}{\partial x_{i}} f_{i}=(\nabla \otimes h) \circ \boldsymbol{f} .
$$

The necessary and sufficient conditions for the transformation of nonlinear system into linear one by feedback are given by the following theorem.

TheOREM 1. Nonlinear system (1) is locally linearizable in the terms of state space and static feedback if and only if in the neighbourhood $U$ of equilibrium point $\boldsymbol{f}\left(\mathbf{x}_{0}\right)=0$ the following conditions are fulfilled

$$
\begin{gathered}
\operatorname{dim}\left(\operatorname{span}\left\{\operatorname{ad}_{\boldsymbol{f}}^{j} \boldsymbol{g}_{i}\right\}\right)=n, 1 \leq i \leq m, j=0, \ldots, n-1, \\
\left.\operatorname{ad}_{\boldsymbol{f}}^{k} \boldsymbol{g}_{i}, \operatorname{ad}_{\boldsymbol{f}}^{l} \boldsymbol{g}_{j}\right] \in \Delta, 1 \leq i, j \leq m, k, l \geq 0 \forall \mathbf{x} \in V .
\end{gathered}
$$

where $g$ is involutive distribution of $(n-1)$ rank. If the conditions (7a) and (7b) are fulfilled, then according to Frobenius theorem [10] there is a scalar function $h(\mathbf{x}): \mathbf{R}^{n} \rightarrow \mathbf{R}$ such that in the neighbourhood of $\mathbf{x}_{0}$ point the following system of equations is satisfied:

$$
\begin{aligned}
& \left\langle d h, \operatorname{ad}_{(-\boldsymbol{f})}^{n-1} \boldsymbol{g}\right\rangle=c \neq 0, \\
& \left\langle d h, \operatorname{ad}_{(-\boldsymbol{f})}^{i} \boldsymbol{g}\right\rangle=0,0 \leq i \leq n-2,
\end{aligned}
$$


and $h(0)=0$.

In other words, conditions (7a) and (7b) of Theorem 1 guarantee that there exists solution $h$ of the system of differential equations

$$
\begin{aligned}
\langle\mathrm{d} h, \boldsymbol{g}\rangle & =0, \\
\left\langle\mathrm{~d} h, \mathrm{ad}_{-\boldsymbol{f}} \boldsymbol{g}\right\rangle & =0, \\
\vdots & \\
\left\langle\mathrm{d} h, \mathrm{ad}_{-\boldsymbol{f}}^{n-2} \mathbf{g}\right\rangle & =0, \\
\left\langle\mathrm{~d} h, \mathrm{ad}_{-\boldsymbol{f}}^{n-1} \mathbf{g}\right\rangle & =c(\mathbf{x}) .
\end{aligned}
$$

Defining $n$ of function $h(\mathbf{x}), L_{\boldsymbol{f}} h(\mathbf{x}), \operatorname{dots} L_{\boldsymbol{f}}^{n-1} h(\mathbf{x})$ we obtain the sought vector of $S(\mathbf{x})$ transformation

$$
S(\mathbf{x})=\left[\begin{array}{c}
S_{1}\left(x_{1}, \ldots, x_{n}\right) \\
S_{2}\left(x_{1}, \ldots, x_{n}\right) \\
\vdots \\
S_{n}\left(x_{1}, \ldots, x_{n}\right)
\end{array}\right]=\left[\begin{array}{c}
z_{1}(t) \\
z_{2}(t) \\
\vdots \\
z_{n}(t)
\end{array}\right]=\left[\begin{array}{c}
h(\mathbf{x}) L_{\boldsymbol{f}} h(\mathbf{x}) \\
\vdots \\
L_{\boldsymbol{f}}^{n-1} h(\vec{x})
\end{array}\right] .
$$

By differentiating (10) with respect to time the linearized system of equations takes the form

$$
\begin{aligned}
& \dot{z}_{i}=z_{i+1}, \quad 1 \leq i \leq n-1, \\
& \dot{z}_{n}=L_{\boldsymbol{f}}^{n} h(\mathbf{x})+L_{\boldsymbol{g}} L_{\boldsymbol{f}}^{n-1} h(\mathbf{x}) \cdot u(t) .
\end{aligned}
$$

After introducing static feedback represented by

$$
\begin{array}{r}
u(t)=-\frac{L_{\boldsymbol{f}}^{n} h(\mathbf{x})}{L_{\boldsymbol{g}} L_{\boldsymbol{f}}^{n-1} h(\mathbf{x})}+\frac{1}{L_{\boldsymbol{g}} L_{\boldsymbol{f}}^{n-1} h(\mathbf{x})} v(t)= \\
\alpha(\mathbf{x})+\beta(\mathbf{x}) v(t),
\end{array}
$$

where $v(t)$ is a new power supply, in the transformed coordinate system $\left(U, z_{1}, z_{2}, \ldots, z_{n}\right)$ with feedback (12) we obtain the following representation of linearized system in the matrix notation:

$$
\left[\begin{array}{c}
\dot{z}_{1} \\
\dot{z}_{2} \\
\dot{z}_{3} \\
\vdots \\
\dot{z}_{n-1} \\
\dot{z}_{n}
\end{array}\right]=\left[\begin{array}{cccccc}
0 & 1 & 0 & \ldots & 0 & 0 \\
0 & 0 & 1 & \ldots & 0 & 0 \\
0 & 0 & 0 & \ldots & 0 & 0 \\
\vdots & \vdots & \vdots & \ddots & \vdots & \vdots \\
0 & 0 & 0 & \ldots & 0 & 1 \\
0 & 0 & 0 & \ldots & 0 & 0
\end{array}\right]\left[\begin{array}{c}
z_{1} \\
z_{2} \\
z_{3} \\
\vdots \\
z_{n-1} \\
z_{n}
\end{array}\right]+\left[\begin{array}{c}
0 \\
0 \\
0 \\
\vdots \\
0 \\
1
\end{array}\right] v(t)
$$

The linear system (13) in the other notation can be written as

$$
\dot{\boldsymbol{z}}(t)=\mathbf{A} \boldsymbol{z}+\boldsymbol{b} \cdot v(t),
$$

where $\mathbf{A}$ is matrix ( $\mathrm{n} \times \mathrm{n}), \boldsymbol{b}$ is a vector of the dimension $(n \times 1)$.

\section{EXAMPLE}

Let us consider an indefinite state in the electrical circuit of the third order containing nonlinear resistor presented in Fig. 1. The assumed current-voltage characteristics of a nonlinear resistive element is

$$
U(i)=a i \ln i,
$$

where $a$ is a coefficient of $(1 \times 1)$ dimension. The equations describing the circuit are

$$
\begin{aligned}
& u_{1}=L_{1} \frac{\mathrm{d} i_{1}}{\mathrm{~d} t}+u(i)-u(t), \\
& u_{2}=L_{2} \frac{\mathrm{d} i_{2}}{\mathrm{~d} t}+R_{2} i_{2} \\
& u_{3}=u_{c}
\end{aligned}
$$

Since $i_{3}=i_{1}-i_{2}, u_{1}=-u_{3}, u_{2}-u_{3}=0$ equations $(16)$ can be written as

$$
\begin{aligned}
\frac{\mathrm{d} i_{1}}{\mathrm{~d} t} & =-\frac{a}{L_{1}} i_{1} \ln i_{1}-\frac{1}{L_{1}} u_{c}+\frac{1}{L_{1}} u(t), \\
\frac{\mathrm{d} i_{2}}{\mathrm{~d} t} & =-\frac{R_{2}}{L_{2}} i_{2}+u_{c}, \\
\frac{\mathrm{d} u_{c}}{\mathrm{~d} t} & =\frac{1}{C} i_{1}-\frac{1}{C} i_{2} .
\end{aligned}
$$

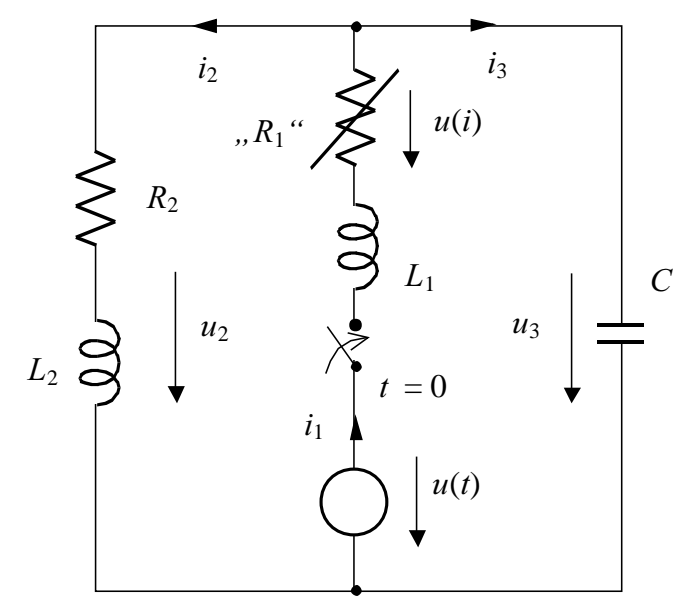

Fig. 1. Diagram of nonlinear electrical circuit of the third order

If we assume that state variables are the currents flowing through the inductor and the capacitor voltage: $i_{1}=x_{1}, i_{2}=x_{2}, u_{c}=x_{3}$ and insert them into (17) then after coefficient normalization the system of equations takes the form

$$
\begin{aligned}
& \dot{x}_{1}=-x_{1} \ln x_{1}-x_{3}+u(t), \\
& \dot{x}_{2}=-x_{2}+x_{3}, \\
& \dot{x}_{3}=x_{1}-x_{2} .
\end{aligned}
$$

Vectors $\boldsymbol{f}(\mathbf{x}), \mathbf{g}(\mathbf{x}),\left(\boldsymbol{f}, \boldsymbol{g} \in \mathbf{R}^{3}, n=3\right)$ in (18) are

$$
\begin{aligned}
& \boldsymbol{f}=\left[\begin{array}{c}
-i_{1} \ln i_{1}-u_{c} \\
-i_{2}+u_{c} \\
i_{1}-i_{2}
\end{array}\right]=\left[\begin{array}{c}
-x_{1} \ln x_{1}-x_{3} \\
-x_{2}+x_{3} \\
x_{1}-x_{2}
\end{array}\right]= \\
& \left(-x_{1} \ln x_{1}-x_{3}\right) \frac{\partial}{\partial x_{1}}+\left(x_{3}-x_{2}\right) \frac{\partial}{\partial x_{2}}+\left(x_{1}-x_{2}\right) \frac{\partial}{\partial x_{3}},
\end{aligned}
$$




$$
\boldsymbol{g}=\left[\begin{array}{l}
1 \\
0 \\
0
\end{array}\right]=\frac{\partial}{\partial x_{1}}
$$

In order to determine the linearizing transformation we should check the conditions in which $S(\mathbf{x})$ transformation exists for system (18) using Theorem 1 . For this purpose, for vector functions $\boldsymbol{f}$ and $\boldsymbol{g}$ we determine a set of vectors (which are Lie brackets) which for a system with a single power supply (input) takes the form

$$
\operatorname{span}\left\{\boldsymbol{g}, \operatorname{ad}_{\boldsymbol{f}}^{1} \boldsymbol{g}, \ldots, \operatorname{ad}_{\boldsymbol{f}}^{n-1} \boldsymbol{g}\right\}=n
$$

In this case we obtain the distribution of vectors

$$
\Delta=\left\{\boldsymbol{g}, \operatorname{ad}_{\boldsymbol{f}}^{1} \boldsymbol{g}, \operatorname{ad}_{\boldsymbol{f}}^{2} \boldsymbol{g}\right\}
$$

By determining next Lie brackets that form distribution $\mathbf{g}$ and are present in $(22)$ we get

$$
\begin{gathered}
\operatorname{ad}_{\boldsymbol{f}} \boldsymbol{g}=\frac{\partial}{\partial \mathbf{x}} \boldsymbol{g} \circ \boldsymbol{f}-\frac{\partial \boldsymbol{f}}{\partial \mathbf{x}} \circ \boldsymbol{g}=\left[\begin{array}{lll}
0 & 0 & 0 \\
0 & 0 & 0 \\
0 & 0 & 0
\end{array}\right]\left[\begin{array}{l}
f_{1} \\
f_{2} \\
f_{3}
\end{array}\right] \\
-\left[\begin{array}{ccc}
-\ln x_{1}-1 & 0 & -1 \\
0 & -1 & 1 \\
1 & -1 & 0
\end{array}\right]\left[\begin{array}{l}
1 \\
0 \\
0
\end{array}\right]=\left[\begin{array}{c}
\ln x_{1}+1 \\
0 \\
-1
\end{array}\right],
\end{gathered}
$$

$$
\operatorname{ad}_{\boldsymbol{f}}^{2} \boldsymbol{g}=\operatorname{ad}_{\boldsymbol{f}}\left(\operatorname{ad}_{\boldsymbol{f}} \boldsymbol{g}\right)=\frac{\partial}{\partial \boldsymbol{x}}\left(\operatorname{ad}_{\boldsymbol{f}} \boldsymbol{g}\right) \circ \boldsymbol{f}-\frac{\partial \boldsymbol{f}}{\partial \mathbf{x}} \circ\left(\operatorname{ad}_{\boldsymbol{f}} \boldsymbol{g}\right)=
$$

$$
\begin{gathered}
{\left[\begin{array}{ccc}
1 / x_{1} & 0 & 0 \\
0 & 0 & 0 \\
0 & 0 & 0
\end{array}\right] \circ\left[\begin{array}{c}
-x_{1} \ln x_{1}-x_{3} \\
-x_{2}+x_{3} \\
x_{1}-x_{2}
\end{array}\right]-} \\
{\left[\begin{array}{ccc}
-\ln x_{1}-1 & 0 & -1 \\
0 & -1 & 1 \\
1 & -1 & 0
\end{array}\right]\left[\begin{array}{c}
\ln x_{1}+1 \\
0 \\
-1
\end{array}\right]=} \\
{\left[\begin{array}{c}
\left(\ln x_{1}\right)^{2}+\ln x_{1}-\frac{x_{3}}{x_{1}} \\
1 \\
-\ln x_{1}-1
\end{array}\right] .}
\end{gathered}
$$

From the computations it follows that

$$
\begin{aligned}
& \operatorname{span}\left(\mathbf{g}, \operatorname{ad}_{\boldsymbol{f}}^{1}, \operatorname{ad}_{\boldsymbol{f}}^{2}\right)= \\
& \left(\left[\begin{array}{l}
1 \\
0 \\
0
\end{array}\right],\left[\begin{array}{c}
\ln x_{1}+1 \\
0 \\
-1
\end{array}\right],\left[\begin{array}{c}
\left(\ln x_{1}\right)^{2}+\ln x_{1}-\frac{x_{3}}{x_{1}} \\
1 \\
-\ln x_{1}-1
\end{array}\right]\right)
\end{aligned}
$$

Hence the distribution $\Delta$ represented by matrix $\mathbf{D}$ has the form

$$
\Delta=\left[\begin{array}{ccc}
1 & \ln x_{1}+1 & \left(\ln x_{1}\right)^{2}+\ln x_{1}-\left(x_{3} / x_{1}\right) \\
0 & 0 & 1 \\
0 & -1 & -\ln x_{1}-1
\end{array}\right]=\mathbf{D}
$$

Since $\operatorname{det} \mathbf{D}=-1 \neq 0$, a nonsingular distribution, the set of vectors $\left\{\boldsymbol{g}, \operatorname{ad}_{\boldsymbol{f}} \boldsymbol{g}, \operatorname{ad}_{\boldsymbol{f}}^{2} \boldsymbol{g}\right\}$ is linearly independent. Thus the condition (7a) of Theorem 1 is fulfilled. Similarly, we should check whether $(7 \mathrm{~b})$ is also satisfied.
Distribution D is involuntive (integrable) when the following relations are met:

$$
\begin{aligned}
{\left[\boldsymbol{g}, \operatorname{ad}_{\boldsymbol{f}} \boldsymbol{g}\right] } & \in \mathbf{D}, \\
{\left[\boldsymbol{g}, \operatorname{ad}_{\boldsymbol{f}}^{2} \boldsymbol{g}\right] } & \in \mathbf{D}, \\
{\left[\operatorname{ad}_{\boldsymbol{f}} \boldsymbol{g} \operatorname{ad}_{\boldsymbol{f}}^{2} \boldsymbol{g}\right] } & \in \mathbf{D},
\end{aligned}
$$

which means that each bracket $\left[X_{i}, X_{j}\right]$ can be represented as a linear combination of vectors $\left\{\mathbf{X}_{1}, \mathbf{X}_{2}, \mathbf{X}_{3}\right\}$, that is

$$
\left[X_{i}, X_{j}\right]=\sum_{l=1}^{3} C_{i j}^{l} \mathbf{x}_{l} \underset{i=1, j=2}{=} C_{i j}^{1} \mathbf{X}_{1}+C_{i j}^{2} \mathbf{x}_{2}+C_{i j}^{3} \mathbf{x}_{3}
$$

When making calculations we can easily check that both relations (27) and the condition (7b) of Theorem 1 are met. Each bracket (27) can be expressed as a linear combination of vectors $\left\{\boldsymbol{g}, \operatorname{ad}_{\boldsymbol{f}} \boldsymbol{g}, \operatorname{ad}_{\boldsymbol{f}}^{2} \boldsymbol{g}\right\}$. Thus, the set of vectors $\mathbf{D}$ is an involuntive, linearly independent set and matrix $\operatorname{rank} \mathbf{D}=3=n$ equals the dimension of the space $\mathbf{R}^{3}=n$. Hence, we seek the function $h(\mathbf{x})$ satisfying the system of equations (8) which for $n=3$ takes the form

$$
\begin{aligned}
\langle\mathrm{d} h, \boldsymbol{g}\rangle & =0, \\
\left\langle\mathrm{~d} h, \mathrm{ad}_{-\mathbf{f}} \mathbf{g}\right\rangle & =0, \\
\left\langle\mathrm{~d} h, \mathrm{ad}_{-\mathbf{f}}^{2} \mathbf{g}\right\rangle & =c \neq 0 .
\end{aligned}
$$

Determining vectors $\operatorname{ad}_{-\boldsymbol{f}} \boldsymbol{g}, \operatorname{ad}_{-\boldsymbol{f}}^{2} \boldsymbol{g}$ successively and substituting them to equations (10) we find that the scalar function $h(x)$ sought for the considered system has the form

$$
h(\mathbf{x})=c x_{2} .
$$

Thus transformation vectors $S(\mathbf{x})$ (10) have the following representation

$$
\begin{aligned}
& S_{1}(\mathbf{x})=h(\mathbf{x})=z_{1}(t) \\
& S_{2}(\mathbf{x})=L_{\boldsymbol{f}}^{1} h(\mathbf{x})=z_{2}(t), \\
& S_{3}(\mathbf{x})=L_{\boldsymbol{f}}^{2} h(\mathbf{x})=z_{3}(t) .
\end{aligned}
$$

Directly from (30) (for $c=1$ ) we can determine the differential of a function $h(\mathbf{x})$ from (6) for Lie derivative of the function $h$ :

$$
\frac{\partial h(\mathbf{x})}{\partial \mathbf{x}}=\left[\begin{array}{lll}
\frac{\partial h(\mathbf{x})}{\partial x_{1}} & \frac{\partial h(\mathbf{x})}{\partial x_{2}} & \frac{\partial h(\mathbf{x})}{\partial x_{3}}
\end{array}\right]=\left[\begin{array}{lll}
0 & 1 & 0
\end{array}\right] .
$$

Derivatives are calculated as follows

$$
L_{\boldsymbol{f}}=\left[\begin{array}{lll}
0 & 1 & 0
\end{array}\right]\left[\begin{array}{c}
-x_{1} \ln x_{1}-x_{3} \\
-x_{2}+x_{3} \\
x_{1}-x_{2}
\end{array}\right]=-x_{2}+x_{3}
$$

$$
\begin{array}{r}
L_{\boldsymbol{f}}^{2} h=L_{\boldsymbol{f}}\left(L_{\boldsymbol{f}} h\right)=\left[\begin{array}{lll}
0 & 1 & 0
\end{array}\right]\left[\begin{array}{c}
-x_{1} \ln x_{1}-x_{3} \\
-x_{2}+x_{3} \\
x_{1}-x_{2}
\end{array}\right]= \\
x_{2}-x_{3}+x_{1}-x_{2}=x_{1}-x_{3} .
\end{array}
$$


The result is that:

$$
\begin{aligned}
& z_{1}(t)=h(\mathbf{x})=x_{2}, \\
& z_{2}(t)=L_{\boldsymbol{f}} h(\mathbf{x})=-x_{2}+x_{3}, \\
& z_{3}(t)=L_{\boldsymbol{f}}^{2} h(\mathbf{x})=x_{1}-x_{3} .
\end{aligned}
$$

After differentiating (31) with respect to time the equation of state in new coordinates takes the form

$$
\begin{aligned}
& \dot{z}_{1}(t)=\dot{x}_{2}=-x_{2}+x_{3}=z_{2}(t) \\
& \dot{z}_{2}(t)=-\dot{x}_{2}+\dot{x}_{3}=x_{2}-x_{3}+x_{1}-x_{2}=x_{1}-x_{3}=z_{3}(t), \\
& \dot{z}_{3}(t)=\dot{x}_{1}-\dot{x}_{3}=-x_{1} \ln x_{1}-x_{3}-x_{1}+x_{2}+u(t) .
\end{aligned}
$$

The last equation of the system (36) is linearized by applying (12). Calculating the derivatives from (12), we obtain

$$
\begin{gathered}
L_{\boldsymbol{f}}^{3} h(\mathbf{x})=L_{\boldsymbol{f}}\left(L_{\boldsymbol{f}}^{2} h(\mathbf{x})\right)=L_{\boldsymbol{f}}\left(x_{1}-x_{3}\right)= \\
{\left[\begin{array}{lll}
1 & 0 & -1
\end{array}\right]\left[\begin{array}{c}
-x_{1} \ln x_{1}-x_{3} \\
-x_{2}+x_{3} \\
x_{1}-x_{2}
\end{array}\right]=} \\
-x_{1} \ln x_{1}-x_{3}-x_{1}+x_{2}, \\
L_{\mathbf{g}} L_{\boldsymbol{f}}^{2} h(\mathbf{x})=L_{\mathbf{g}}\left(x_{1}-x_{3}\right)=\left[\begin{array}{lll}
1 & 0 & -1
\end{array}\right]\left[\begin{array}{l}
1 \\
0 \\
0
\end{array}\right]=1 .
\end{gathered}
$$

Hence

$$
\begin{aligned}
& \alpha(\mathbf{x})=-\frac{L_{\boldsymbol{f}}^{3} h(\mathbf{x})}{L_{\boldsymbol{g}} L_{\boldsymbol{f}}^{2} h(\mathbf{x})}=-x_{1} \ln x_{1}-x_{3}-x_{1}+x_{2}, \\
& \beta(\mathbf{x})=\frac{1}{L_{\boldsymbol{g}} L_{\boldsymbol{f}}^{2} h(\mathbf{x})}=1 .
\end{aligned}
$$

Thus according to (12) a new rule decomposing inputstate of nonlinear system is defined by

$$
u(t)=x_{1} \ln x_{1}+x_{3}+x_{1}-x_{2}-v(t) .
$$

Hence, a new power supply

$$
v(t)=-x_{1} \ln x_{1}-x_{3}-x_{1}+x_{2}+u(t) .
$$

For such feedback the dynamics of linearized system in the new coordinates can be written as follows:

$$
\begin{aligned}
& \dot{z}_{1}(t)=z_{2}(t), \\
& \dot{z}_{2}(t)=z_{3}(t), \\
& \dot{z}_{3}(t)=v(t) .
\end{aligned}
$$

where $v(t)$ is a new power supply after transformation of supply $u(t)$.

The resulting system of equations is a linear system in the canonical form (13), decomposed and decoupled. Each state variable is determined by differential equation with the right-hand side expressed by the simplest form of the first degree polynomial.

\section{RESULTS VERIFICATION}

In order to verify the results obtained, the numerical simulation of the solution of nonlinear equation of state (18) and its linearized model (43) was conducted. The first stage was a digital simulation of non-linear differential equations representing the model of nonlinear electrical circuit shown in Fig. 1. The simulation was carried out for zero initial conditions: $x_{1}(0)=0, x_{2}(0)=0$, $x_{3}(0)=0$ and input $u(t)=\mathbf{1}(t)$. The results in the form of time characteristics of state variables and phase trajectory are presented in Fig. 2.

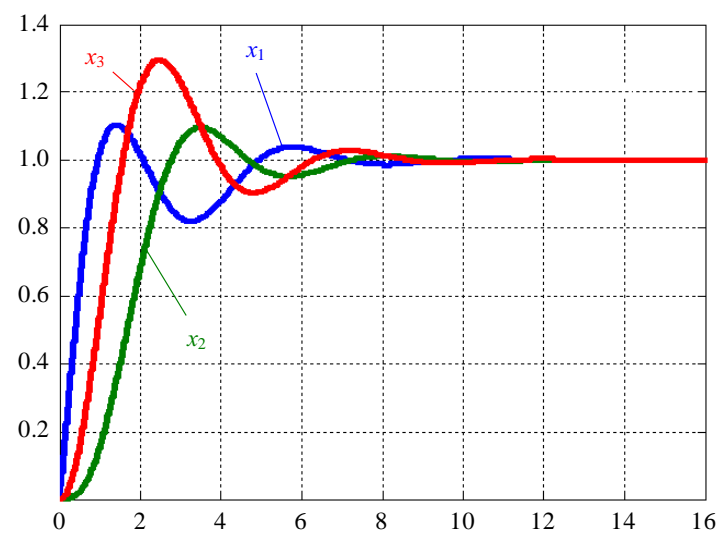

Fig. 2. Numerical solution of nonlinear system of equations (18)

In order to conduct simulation for a linearized system described by system of equations (43) computations were carried out. From relation (35) the state variables $\mathbf{x}$ :

$$
\begin{aligned}
& x_{1}(t)=z_{3}(t)-z_{1}(t)-z_{2}(t), \\
& x_{2}(t)=z_{1}(t) \\
& x_{3}(t)=z_{1}(t)+z_{2}(t) .
\end{aligned}
$$

together with new power supply are determined:

$$
v(t)=-\frac{b^{2}}{C} u-\frac{b}{C} z_{2}(t)+\frac{b}{C}\left(z_{1}(t)\right)^{2} .
$$

By substituting the above relations to linear equation of state (43) the characteristics of state variables for the original was determined. The initial conditions were determined from the following relations.

$$
\begin{aligned}
& z_{1}(0)=x_{2}(0) \\
& z_{2}(0)=-x_{2}(0)+x_{3}(0), \\
& z_{3}(t)=x_{1}(0)-x_{3}(0) .
\end{aligned}
$$

In Fig. 3 and Fig. 4, the time course of the new supply $v(t)$ and are the phase trajectory of the solution are presented. 


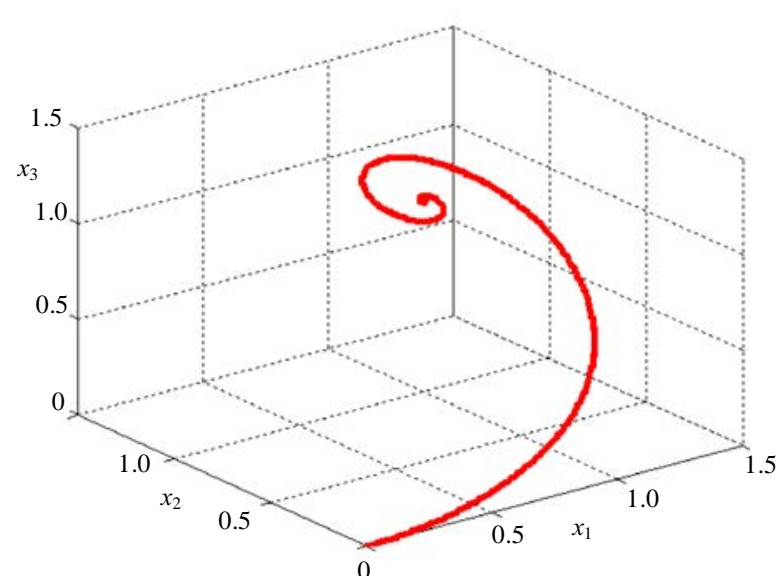

Fig. 3. The time characteristic of the new supply $v(t)$

\section{CONCLUSION}

Numerical solutions (time characteristics) of state equations of nonlinear systems and the corresponding solutions of linearized systems are the same which proves that the derived mathematical models of linear systems subjected to linearization are correct. It means that nonlinear system can be simulated by linearized system.

It appears that linearization of the system under consideration is a combination of transformation of state variables and input transformation. The above example shows that in some cases, in order to solve the problem, a combination of the transformation of state variables and input transformation by feedback should be used. It is worth adding that nonlinear systems described by the equation in controllable canonical form (as in the case of equation 36) written in the following general form

$$
\left[\begin{array}{c}
\dot{x}_{1} \\
\vdots \\
\dot{x}_{n-1} \\
\dot{x}_{n}
\end{array}\right]=\left[\begin{array}{c}
x_{2} \\
\vdots \\
x_{n} \\
f(\mathbf{x})+g(\mathbf{x}) u
\end{array}\right]
$$

are often mathematical models of numerous electrical circuits of the nth order. As an example, non-linear RLC circuit or controllable generator circuits [31] described by mathematical models by Van der Pol equation can be given. For such systems linearization by feedback can be used directly by the introduction of a new power supply removing non-linearity. Linearization of nonlinear mathematical models of dynamic systems by means of feedback can be successfully applied in practical problems which is confirmed by the case of nonlinear electrical circuit. Undoubtedly, the presented theory can also be used, for example, in the automatic control of complex electrical and electronic equipment and electric drives.

\section{REFERENCES}

[1] LAMBERT, J. D.: Numerical Methods for Ordinary Differential Systems, John Wiley \& Sons, Chichester, 1991.

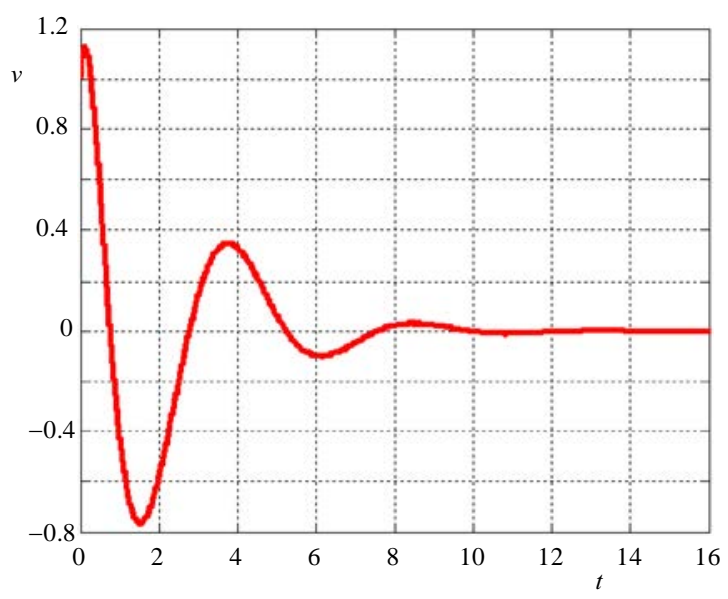

Fig. 4. Phase trajectory of the solution of non-linear system of equations (18)

[2] QUARTERONI, A.-SACCO, R.-SALERI, F.: Numerical Mathematics, Springer-Verlag, New York, 2000.

[3] ATKInSON, K.-HAN, W.-STEWART, D. : Numerical Solution of Ordinary Differential Equations, John Wiley \& Sons, Inc., Hoboken, New Jersey, 2009.

[4] NAJM, F. N.: Circuit Simulation, John Wiley \& Sons, Inc., Hoboken, 2010.

[5] BROCKETT, R. W.: Nonlinear Systems and Differential Geometry, Proc. Of IEEE 64 No. 1 (1976), 61-71.

[6] JAKUBCZYK, B.-RESPONDEK, W. : On Linearization of Control Systems, Bull. Acad. Polonaise, Sci., Ser. Sci. Math. 28 (1980), 517-522.

[7] BYRNES, C. I.-ISIDORI, A. : Local Stabilization of Minimum Phase Nonlinear Systems, Syst. Control Lett. 11 (1988), 9-19.

[8] CELIKOVSKY, S.-NIJMEIJER, H. : Syst. Control Lett. 27 No. 3 (1996), 135-144.

[9] JORDAN, A.-NOWACKI, J. P.: Global Linearization of Non-Linear State Equations, International Journal Applied Electromagnetics and Mechanics 19 (2004), 637-642.

[10] NIJMEIJER, H.—van der SCHAFT, A. J. : Nonlinear Dynamical Control Systems, Springer-Verlag, New York, 1991.

[11] ISIDORI, A.: Nonlinear Control Systems: An Introduction, Springer, Berlin, 1989.

[12] ISIDORI, A. : Nonlinear Control Systems, Springer, Berlin, 1995.

[13] BROCKETT, R. W.: Feedback Invariants for Nonlinear Systems, Proc. 6th IFACWorld Congr., vol. 6, Helsinki, 1978, pp. $1115-1120$.

[14] ISIDORI, A.-KRENER, A.-GORI, A. J.-MONACO, S. : Nonlinear Decoupling via Feedback: a Differential Geometric Approach, IEEE Trans. Automat. Contr. AC-26 No. 2 (1981), $331-345$.

[15] TALL, I. A.-RESPONDEK, W.: Feedback Linearizable Strict Feedforward Systems, in Proceedings of the 47th IEEE Conference on Decision and Control, Canc'un, Mexico, 2008, pp. 2499-2504.

16] DEVANATHAN, R.: Linearization Condition through State Feedback, IEEE Transactions on Automatic Control 46 No. 8 (2001), 1257-1260.

[17] BOUKAS, T. K.-HABETLER, T. G. : High-Performance Induction Motor Speed Control using Exact Feedback Linearization with State and State Derivative Feedback, IEEE Transactions on Power Electronics 19 No. 4 (2004), 1022-1028.

[18] DEUTSCHER, J.-SCHMID, C.: A State Space Embedding Approach to Approximate Feedback Linearization of Single In- 
put Nonlinear Control Systems, International Journal of Circuit Theory and Applications 16 No. 9 (2006), 421-440.

[19] LÓPEZ-ARAUJO, D. J.-ZAVALA-RO, A.-SANTIBÁÑEZ, V.-REYES, F.: Output-Feedback Adaptive Control for the Global Regulation of Robot Manipulators with Bounded Inputs, International Journal of Control, Automation, and Systems 11 No. 1 (Feb 2013), 105-115.

[20] JORDAN, A.-KACZOREK, T.-MYSZKOWSKI, P.: Linearization of Nonlinear Differential Equations, Published by Bialystok University of Technology, Bialystok, 2007. (in Polish)

[21] JORDAN, A. J.: Linearization of Non-Linear State Equation, Bulletin of The Polish Academy of Sciences. Technical Sciences 54 No. 1 (2006), 63-73.

[22] ELWAKIL, A.-KENNEDY, M. : Construction of Classes of Circuit-Independent Chaotic Oscillators using Passive-Only Nonlinear Devices, IEEE Transactions on Circuits and Systems-I 48 No. 3 (2001), 289-307.

[23] ZAWADZKI, A.-RÓŻOWICZ, S.: Application of Input State of the System Transformation for Linearization of some Nonlinear Generators, International Journal of Control, Automation, and Systems 13 No. 3 ( June 2015), (accepted for publication).

[24] ZAWADZKI, A.: Application of Local Coordinates Rectification in Linearization of Selected Parameters of Dynamic Nonlinear Systems, COMPEL - The International Journal for Computation and Mathematics in Electrical and Electronic Engineering 33 No. 5 (2014), 1819-1830.

[25] BOURBAKI, N. : Lie Groups and Lie Algebras, (Chapters 1-3), Springer, Berlin 1998, Lie groups and Lie algebras (Chapters 4-6), Springer, Berlin, 2002.

[26] BUMP, D.: Lie Groups, Graduate Texts in Mathematics, vol. 225, Springer, New York, 2004.

[27] SERRE, J. P.: Complex Semisimple Lie Algebras, Springer-Verlag, Berlin, 2001.
[28] YAT-SUN, P.-WAI-YIN, P.: Application of Elementary Differential Geometry to Influence Analysis, International Press of Boston Inc, Boston, 2012.

[29] BODSON, M.-CHIASSONS, J.: Differential-Geometric Methods for Control of Electric Motors, International Journal of Circuit Theory and Applications 8 No. 11 (1998), 923-954.

[30] De LUCA, A.: Decoupling and Feedback Linearization of Robots with Mixed Rigid/Elastic Joints, Int. J. Robust Nonlinear Control 8 No. 11 (1998), 965-977.

[31] MURRAY WONHAM, W.: Linear Multivariable Control. A Geometric Approach, Springer-Verlag, New York, 1985.

Received 10 October 2015

Andrzej Zawadzki has received his MSc at Kielce University of Technology in 1988 and $\mathrm{PhD}$ at Warsaw University of Technology in 2001. From 2001 he has been working as lecturer at Department of Electrical Devices and Lighting Techniques at Kielce University of Technology, Kielce, Poland. His scientific interests contain: electrical circuits theory with special focus on nonlinear systems and their transformation into linear forms, modeling physical processes and systems with the use of differential calculus of fractional order.

Sebastian Różowicz has received his MSc at Kielce University of Technology in 2002 and $\mathrm{PhD}$ at Kielce University of Technology in 2012. From 2012 he has been working as Assistant Professor at Department of Power Electronics at Kielce University of Technology, Poland. His scientific interests contain: modeling physical processes and systems with the use of differential calculus in the automotive engineering, analysis of the spark discharge energy and mathematical models of ignition systems. 